\title{
Accurate first-principle equation of state for the One-Component Plasma
}

\author{
Nikolai V. Brilliantov ${ }^{1,2}$ \\ ${ }^{1}$ Chemical Physics Theory Group, Department of Chemistry, University of Toronto, Toronto, \\ Canada M5S $3 \mathrm{H} 6$ \\ ${ }^{2}$ Moscow State University, Physics Department, Moscow 119899, Russia
}

\begin{abstract}
Accurate "first-principle" expressions for the excess free energy $F_{\text {ex }}$ and internal energy $U_{\text {ex }}$ of the classical one-component plasma (OCP) are obtained. We use the Hubbard-Schofield transformation that maps the OCP Hamiltonian onto the Ising-like Hamiltonian, with coefficients expressed in terms of equilibrium correlation functions of a reference system. We use the ideal gas as a reference system for which all the correlation functions are known. Explicit calculations are performed with the high-order terms in the Ising-like Hamiltonian omitted. For small values of the plasma parameter $\Gamma$ the DebyeHuckel result for $F_{\text {ex }}$ and $U_{\text {ex }}$ is recovered. For $\Gamma \gg 1$, these depend linearly on $\Gamma$ in accordance with the Monte Carlo findings for the OCP. The MC data for the internal energy are reproduced fairly well by the obtained analytical expression.
\end{abstract}

\section{INTRODUCTION}

The one component plasma (OCP) model is one of the basic models in the condensed matter physics [1,2]. Besides of its direct astrophysical applications [2], to model the ionized 
matter in white dwarfs [3], outer layers of neutron stars and interiors of heavy planets 幽,5], OCP is widely used as a reference model for a variety of systems, ranging from alkali metals [6] to colloidal solutions [9]1]. The application of the OCP is not restricted by classical systems; it is also used when quantum effects are important [12].

The OCP model is formulated as a system of point particles, interacting via the Coulombic potential, which move in a uniform neutralizing background [1,2]. All thermodynamic properties of the OCP depend only on the dimensionless plasma parameter $\Gamma=l_{B} / a_{c}$, where $l_{B}=e^{2} / k_{B} T$ is the Bjerrum length ( $e$ is the charge of the particles, $k_{B}$ is the Boltzmann's constant, $T$ is the temperature) and $a_{c}=(3 / 4 \pi \rho)^{1 / 3}$ is the ion-sphere radius with $\rho=N / \Omega$ being the concentration of particles ( $N$ is the number of particles, $\Omega$ is the volume of the system).

For small values of $\Gamma$, which correspond to a hot and/or dilute system, the Debye-Huckel theory accurately describes the thermodynamic properties of the OCP. For the excess free energy and internal energy this gives in the $\Gamma \rightarrow 0$ limit:

$$
\frac{F_{\mathrm{ex}}}{k_{B} T N}=-\frac{1}{\sqrt{3}} \Gamma^{3 / 2} ; \quad \frac{U_{\mathrm{ex}}}{k_{B} T N}=-\frac{\sqrt{3}}{2} \Gamma^{3 / 2}
$$

Abe expansion [13 for the classical OCP provides next terms for that limit (in the present study we consider the classical case):

$$
\begin{aligned}
\frac{F_{\mathrm{ex}}}{k_{B} T N} & =-\frac{1}{\sqrt{3}} \Gamma^{3 / 2}-\frac{c}{3} \Gamma^{3}-\frac{1}{8} \Gamma^{3}(3 \log \Gamma-1)+\cdots \\
\frac{U_{\mathrm{ex}}}{k_{B} T N} & =-\frac{\sqrt{3}}{2} \Gamma^{3 / 2}-\left(c+\frac{3}{8}\right) \Gamma^{3}-\frac{3}{8} \Gamma^{3}(3 \log \Gamma-1)+\cdots
\end{aligned}
$$

where $c=\frac{9}{8} \log 3+\frac{3}{2} \gamma-1=1.101762 \ldots$, and $\gamma$ is the Euler's constant. The analytical Abe expansion seems to be fairly accurate for $\Gamma$ up to 0.1 [14]. The next few terms for the $\Gamma \rightarrow 0$ expansion calculated in Ref. [15] allow to use the small- $\Gamma$ expansion up to $\Gamma \leq 0.4$.

For larger values of $\Gamma$ the OCP was studied numerically, by means of integral equations, such as Percus-Yevick, hypernetted-chain equations [16], and (most successively) by modified hypernetted-chain equation [17]. Extensive numerical studies have been performed 
by Monte Carlo (MC) [5, 14, 18 21] and Molecular Dynamics 22] technique. To fit available "experimental" data for the excess thermodynamic functions simple analytical fits were proposed [23]:

$$
\begin{aligned}
& \frac{U_{\mathrm{ex}}}{k_{B} T N}=A \Gamma+B \Gamma^{s}+C \\
& \frac{F_{\mathrm{ex}}}{k_{B} T N}=A \Gamma+\frac{B}{s} \Gamma^{s}+(3+C) \log \Gamma-D
\end{aligned}
$$

with $A=-0.8992, B=0.596, s=0.3253, C=-0.268$ and $D=A+B / s+1.1516$. Eqs.(四) and (5) are fairly accurate for the interval $1 \leq \Gamma \leq 220$ [23], but unfortunately they do not give a correctly behavior at $\Gamma \rightarrow 0$. Pade approximants for the $U_{\text {ex }}$ suggested in 24,25 remedy the failure of (田) at small $\Gamma$; one thus obtains very precise description for the whole interval $0 \leq \Gamma \leq 200[25]$.

To describe the limit of very large $\Gamma$ a perturbation theory was proposed [26]; it agrees well with the $\mathrm{MC}$ data for large $\Gamma$, but also lack the proper small- $\Gamma$ behavior. The correct DebyeHuckel behavior at $\Gamma \rightarrow 0$ together with a reasonable $\sim 10 \%$ accuracy for $0 \leq \Gamma \leq 100$ has been obtained in a simple semiphenomenological "Debye-Huckel plus hole" (DHH) theory [27]; it fails, however, for $\Gamma>125$ [27]. A modified DHH theory proposed recently [28] using only one fitting parameter accurately reproduces the $\mathrm{MC}$ results for the large values of $\Gamma$ $(1<\Gamma<200)$ and demonstrates a correct behavior at $\Gamma \rightarrow 0$.

Thus, up to now, no "first-principle" theory of OCP exists which describes accurately the thermodynamic properties in the whole range of $\Gamma$ from the Debye-Huckel limit $\Gamma \rightarrow 0$ up to $\Gamma \gg 1$ limit, where the Wigner crystallization [5] occurs. In the present study we report a simple "first-principle" equation of state for the OCP which has the correct Debye-Huckel behavior for small $\Gamma$ and demonstrates a linear dependence on $\Gamma$ for $\Gamma \gg 1$. It reproduces within $1-2 \%$ accuracy the experimental data for the most of range of $\Gamma(0 \leq \Gamma \leq 250)$ and has a typical deviation of the order of $2-5 \%$ (with a maximal one $\approx 8 \%$ ) for $0.01<\Gamma<1$. The rest of the paper is organized as follows: in the next Sec.II we consider the HubbardSchofield transformation that maps the OCP Hamiltonian onto the Ising-like Hamiltonian and calculate the coefficients of the effective Hamiltonian. In this section we also present the 
field-theoretical formulation for the statistical sum of the OCP which directly follows from the transformation used. Within the Gaussian approximation for the effective Hamiltonian we derive the equation of state for the OCP. In Sec.III we discuss the equation of state obtained and compare the analytical results for the internal excess free energy with the available Monte Carlo data for the OCP. In the last Sec.IV we summarize our findings.

\section{EFFECTIVE HAMILTONIAN AND EQUATION OF STATE FOR THE OCP}

We start from the OCP Hamiltonian which may be written as follows $\left(\beta^{-1}=k_{B} T\right)$ :

$$
H=\frac{1}{2} \beta^{-1} \sum_{\mathbf{k}}^{\prime} \nu_{k}\left(\rho_{\mathbf{k}} \rho_{-\mathbf{k}}-\rho\right)+H_{\mathrm{id}}
$$

where the first term in the right-hand side of Eq.([6) refers to the Coulombic interactions, written in terms of the collective variables,

$$
\rho_{\mathbf{k}}=\frac{1}{\sqrt{\Omega}} \sum_{j=1}^{N} e^{-i \mathbf{k r}_{j}}
$$

where $\mathbf{r}_{j}$ denotes coordinate of $j$-th particle, $\nu_{k}=4 \pi l_{B} / k^{2}$ and $H_{i d}$ is the ideal-gas part of the

Hamiltonian. Summation in Eq.(6) is to be performed over the wave-vectors $\mathbf{k}=\left\{k_{x}, k_{y}, k_{z}\right\}$ with $k_{i}=\frac{2 \pi}{L} l_{i}(i=x, y, z)$, where $l_{i}$ are integers, $L^{3}=\Omega$, and the prime over the sum denotes that the term with $\mathbf{k}=0$ is excluded [29].

\section{A. Hubbard-Schofield transformation}

The configurational integral may be then written in terms of the configurational integral of the reference (ideal gas) system $Q_{R}$ [30,31] as

$$
Q=\left\langle\exp \left\{-\frac{1}{2} \sum_{\mathbf{k}}^{\prime} \nu_{k}\left(\rho_{\mathbf{k}} \rho_{-\mathbf{k}}-\rho\right)\right\}\right\rangle_{R} Q_{R}
$$

where $\langle(\cdots)\rangle_{R}=Q_{R}^{-1} \int d \mathbf{r}^{N}(\cdots)$ denotes the averaging over the reference system. In accordance with the Hubbard-Schofield scheme [30 we use the identity, 


$$
\exp \left(-\frac{1}{2} a^{2} x^{2}\right)=\left(2 \pi a^{2}\right)^{-1 / 2} \int_{-\infty}^{+\infty} \exp \left(-\frac{1}{2} y^{2} / a^{2}+i x y\right) d y
$$

and arrive after some algebra at:

$$
Q=Q_{R} \int \prod_{\mathbf{k}}^{\prime} c_{\mathbf{k}} d \varphi_{\mathbf{k}} \exp \left\{-\frac{1}{2} \sum_{\mathbf{k}}^{\prime} \nu_{k}^{-1} \varphi_{\mathbf{k}} \varphi_{-\mathbf{k}}\right\}\left\langle\exp \left\{i \sum_{\mathbf{k}}^{\prime} \rho_{\mathbf{k}} \varphi_{-\mathbf{k}}\right\}\right\rangle_{R}
$$

where $c_{\mathbf{k}}=\left(2 \pi \nu_{k}\right)^{-1 / 2} e^{\frac{1}{2} \nu_{k} \rho}$, and where the integration is to be performed under the restriction, $\varphi_{-\mathbf{k}}=\varphi_{\mathbf{k}}^{*}\left(\varphi_{\mathbf{k}}^{*}\right.$ is the complex conjugate of $\left.\varphi_{\mathbf{k}}\right)$ [32]. Applying the cumulant theorem [33 to the factor $\left\langle\exp \left\{i \sum_{\mathbf{k}}{ }^{\prime} \rho_{\mathbf{k}} \varphi_{-\mathbf{k}}\right\}\right\rangle_{R}$ one obtains:

$$
\begin{aligned}
& Q=Q_{R} \int \prod_{\mathbf{k}}^{\prime} c_{\mathbf{k}} d \varphi_{\mathbf{k}} e^{-\mathcal{H}}, \text { with } \\
& \mathcal{H}=\sum_{n=2}^{\infty} \Omega^{1-\frac{n}{2}} \sum_{\mathbf{k}_{1}, \ldots \mathbf{k}_{n}}{ }^{\prime} u_{n}\left(\mathbf{k}_{1}, \ldots \mathbf{k}_{n}\right) \varphi_{\mathbf{k}_{1}} \cdots \varphi_{\mathbf{k}_{n}} \\
& u_{2}\left(\mathbf{k}_{1}, \mathbf{k}_{2}\right)=\frac{1}{2} \delta_{\mathbf{k}_{1}+\mathbf{k}_{2}, \mathbf{0}}\left\{\frac{k_{1}^{2}}{4 \pi l_{B}}+\left\langle\rho_{\mathbf{k}_{1}} \rho_{-\mathbf{k}_{1}}\right\rangle_{c R}\right\} \\
& u_{n}\left(\mathbf{k}_{1}, \ldots \mathbf{k}_{n}\right)=-i^{n} \frac{\Omega^{\frac{n}{2}-1}}{n !}\left\langle\rho_{\mathbf{k}_{1}} \ldots \rho_{\mathbf{k}_{n}}\right\rangle_{c R} \quad n>2
\end{aligned}
$$

here $\langle\ldots\rangle_{c R}$ denotes cumulant average [33] for the reference ideal-gas system. As it follows from Eqs.(10), $Q$ is written in a similar way as the partition function for the magnetic system having the Ising-like Hamiltonian [34], where $\varphi_{\vec{k}}$ are the Fourier components of the "spin-field", $\varphi(\vec{r})$. Note that the coefficients of the effective Hamiltonian (10) are expressed in terms of the correlation functions of the ideal-gas system, which are perfectly known (e.g. [35]):

$$
\Omega^{\frac{n}{2}-1}\left\langle\rho_{\mathbf{k}_{1}} \ldots \rho_{\mathbf{k}_{n}}\right\rangle_{c R}=\rho \delta_{\mathbf{k}_{1}+\cdots \mathbf{k}_{n}, \mathbf{0}}
$$

This yields the effective Hamiltonian:

$$
\begin{aligned}
& \mathcal{H}=\frac{1}{2} \sum_{\mathbf{k}}^{\prime}\left[\rho+\left(4 \pi l_{B}\right)^{-1} k^{2}\right] \varphi_{\mathbf{k}} \varphi_{-\mathbf{k}}-\sum_{n=3}^{\infty} \frac{i^{n} \Omega^{1-\frac{n}{2}}}{n !} \rho \sum_{\mathbf{k}_{1}, \ldots \mathbf{k}_{n}}^{\prime} \varphi_{\mathbf{k}_{1}} \cdots \varphi_{\mathbf{k}_{n}} \delta_{\mathbf{k}_{1}+\cdots \mathbf{k}_{n}, \mathbf{0}} \\
& =\mathcal{H}_{G}+\mathcal{H}_{1}
\end{aligned}
$$

where we write explicitly the Gaussian part $\mathcal{H}_{G}$ : 


$$
\mathcal{H}_{G}=\frac{1}{2} \sum_{\mathbf{k}}^{\prime}\left[\rho+\left(4 \pi l_{B}\right)^{-1} k^{2}\right] \varphi_{\mathbf{k}} \varphi_{-\mathbf{k}}
$$

Since all the coefficients of the effective Hamiltonian are known, one can develop the usual perturbation expansion, with $\mathcal{H}_{G}$ being a reference part of the Hamiltonian and with $\mathcal{H}_{1}$ being perturbation (e.g. 34, 36,37).

A simple structure of the effective Hamiltonian $\mathcal{H}$, Eq.(12) suggests a simple, closedform field-theoretical formulation for the partition sum of the OCP, akin the Sine-Gordon representation of the Coulombic gas [38], or the restricted primitive model of electrolytes 36, 39.

\section{B. Field-theoretical model for the OCP}

Using the space-dependent field $\varphi(\mathbf{r})$

$$
\varphi(\mathbf{r})=\frac{1}{\sqrt{\Omega}} \sum_{\mathbf{k}} \varphi_{\mathbf{k}} e^{-i \mathbf{k r}}
$$

It is easy to show that under this transformation the terms in Eq.(12) containing products $\varphi_{\mathbf{k}_{1}} \cdots \varphi_{\mathbf{k}_{n}}$ give rise to the terms $\int d \mathbf{r} \varphi^{n}(\mathbf{r})$, the terms $k^{2} \varphi_{\mathbf{k}} \varphi_{-\mathbf{k}}$ give rise to the term $\int d \mathbf{r}(\nabla \varphi)^{2}$, so that one can write

$$
\mathcal{H}=\rho \int d \mathbf{r}\left[\frac{1}{2}\left(4 \pi l_{B} \rho\right)^{-1}(\nabla \varphi)^{2}-\sum_{n=2}^{\infty} \frac{i^{n}}{n !} \varphi^{n}\right]
$$

and recognize the expansion of $e^{i \varphi}$.

Integration over the Fourier-components $\varphi_{\mathbf{k}}$ in Eq.(10) converts into "field"-integration

over the field $\varphi(\mathbf{r})$. It may be shown that the Jacobian of this transformation does not depend on $\varphi(\mathbf{r})$ and appears as a normalization constant.

Noticing that

$$
\prod_{\mathbf{k}}^{\prime}\left(2 \pi \nu_{k}\right)^{-\frac{1}{2}}=\left[\int \prod_{\mathbf{k}}^{\prime} d \varphi_{\mathbf{k}} \exp \left\{-\frac{1}{2} \sum_{\mathbf{k}}^{\prime} \frac{k^{2}}{4 \pi l_{B}} \varphi_{\mathbf{k}} \varphi_{-\mathbf{k}}\right\}\right]^{-1}
$$

(with the restriction $\varphi_{\mathbf{k}}^{*}=\varphi_{-\mathbf{k}}$ ) and that

$$
\prod_{\mathbf{k}}^{\prime} e^{\frac{1}{2} \nu_{k} \rho}=\exp \left\{\rho \Omega \frac{U(0)}{2 k_{B} T}\right\}
$$


where $U(0)$ is the so-called "self-energy" 38,40, one arrives after some algebra at the fieldtheoretical expression of the statistical sum $Z_{O C P}$ of the OCP:

$$
e^{-\beta F} \equiv Z_{O C P}=\frac{\int \mathcal{D} \varphi \exp \{-\mathcal{H}(\varphi)\}}{\int \mathcal{D} \varphi \exp \left\{-\int d \mathbf{r}\left[\frac{1}{2} \kappa_{D}^{-2}(\nabla \varphi)^{2}-\beta \tilde{\mu}\right]\right\}}
$$

where $\mathcal{D} \varphi$ denotes the "field"-integration,

$$
\mathcal{H}=\int d \mathbf{r}\left[\frac{\kappa_{D}^{-2}}{2}(\nabla \varphi)^{2}+i \varphi-e^{i \varphi}\right]
$$

$\kappa_{D}^{2}=4 \pi l_{B} \rho=4 \pi e^{2} \beta \rho$ is the inverse Debye screening length and

$$
\tilde{\mu}=\mu_{\mathrm{id}}+k_{B} T-U(0) / 2 .
$$

Here $\beta \mu_{\text {id }}=\log \left(\Lambda^{3} / \Omega\right)$ is the ideal-gas chemical potential $\left(\Lambda=h /\left(2 \pi m k_{B} T\right)^{1 / 2}\right.$ is the thermal wavelength). Deriving Eq.(15) we write the statistical sum of the ideal gas as $Z_{\text {id }}=\exp \left[-N \log \left(\Lambda^{3} / \Omega\right)\right]=\exp \left\{-\beta \mu_{\text {id }} \rho \int d \mathbf{r}\right\}$ and rescale the length $\rho d \mathbf{r} \rightarrow d \mathbf{r}$.

As it follows from Eq.(15) the potential function of the effective field-theoretical Hamiltonian for the OCP reads $V(\varphi)=i \varphi-e^{i \varphi}$. This may be compared with the potential function $V_{S G}(\varphi)=\cos \varphi$ of the Sine-Gordon model for the Coulombic gas [38]. Note, that all imaginary terms in Eq.(15) vanish after the field integration due to the symmetry properties of the Hamiltonian under the transformation $\varphi \rightarrow-\varphi$.

Consider now the "saddle-point" approximation to the numerator in Eq.(15). The equation for the "extremal" field which minimizes the effective Hamiltonian reads:

$$
\nabla^{2} \varphi=\kappa_{D}^{2}\left(i-i e^{i \varphi}\right)
$$

Under transformation $\varphi=i e^{2} \phi / k_{B} T$ Eq.(18) converts into

$$
\nabla^{2} \phi=-4 \pi \rho\left[e^{-e \phi / k_{B} T}-1\right]
$$

which is the usual mean-field Poisson-Boltzmann equation for the OCP. This is not surprising since the "saddle-point" approximation is essentially the mean-field one. 


\section{Equation of state for the OCP}

Now we concentrate on the Gaussian part of the effective Hamiltonian and show that even neglecting the non-Gaussian contribution to the effective Hamiltonian, one can obtain fairly accurate equation of state for the OCP, provided that a correct value of the ultraviolet cutoff in the $\mathbf{k}$-space is used. The Gaussian approximation to $\mathcal{H}$ corresponds actually to the Random Phase, or Debye-Hückel approximation, (e.g. 41]). Using $\mathcal{H}_{G}$, Eq.(13) and performing (Gaussian) integration (e.g. 38]) over $\varphi_{\mathbf{k}}$ in (10), one easily finds for the excess free energy of the OCP:

$$
-\beta F_{\mathrm{ex}}=\log \left(Q / Q_{R}\right)=\frac{1}{2} \sum_{\mathbf{k}}^{\prime}\left[\rho \nu_{k}-\log \left(1+\rho \nu_{k}\right)\right]
$$

We argue that the summation in Eq.(20) should be carried out over a finite number of the wave-vectors k. In this we follow the Debye theory of the specific heat of solids (e.g. [42]). Namely, we assume that the total number of degrees of freedom in the system, $3 N$, should be equal to the total number of physically different modes with the wave-vectors $\mathbf{k}$ within the spherical shell of radius $k_{0}$ in the $\mathbf{k}$-space. The number of modes is twice the number of the wave-vectors, since for each $\mathbf{k}$ one has a sine and cosine mode (the amplitude of the k-th mode is a complex number) [43]. Thus we obtain:

$$
2 \frac{\Omega}{8 \pi^{3}} 4 \pi \int_{0}^{k_{0}} k^{2} d k=3 N
$$

where the factor $\Omega / 8 \pi^{3}$ appears when the integration in $\mathbf{k}$-space is used instead of summation. From Eq.(21) follows that $k_{0}=\left(9 \rho \pi^{2}\right)^{1 / 3}$. A similar Debye-like scheme to find the cutoff $k_{0}$ was first proposed for plasma in [44], where somewhat different value of the cutoff wave-vector was reported. Using the $k_{0}$ obtained we write:

$$
\begin{aligned}
-\frac{\beta F_{\mathrm{ex}}}{N} & =\frac{1}{2} \frac{\Omega}{8 \pi^{3}} \frac{4 \pi}{N} \int_{0}^{k_{0}} k^{2} d k\left[\log \left(1+\frac{\kappa_{D}^{2}}{k^{2}}\right)-\frac{\kappa_{D}^{2}}{k^{2}}\right] \\
& =\frac{9}{4} \int_{0}^{1} x^{2} d x\left[\log \left(1+\frac{b \Gamma}{x^{2}}\right)-\frac{b \Gamma}{x^{2}}\right]
\end{aligned}
$$

where $b=\frac{2}{3}\left(\frac{2}{\pi^{2}}\right)^{1 / 3}$. The last integral is easily calculated to obtain for the free energy 


$$
\frac{F_{\mathrm{ex}}}{k_{B} T N}=\frac{3}{4}[\log (1+b \Gamma)-b \Gamma]-\frac{3}{2}(b \Gamma)^{\frac{3}{2}} \arctan \left(\frac{1}{\sqrt{b \Gamma}}\right)
$$

and for the internal energy:

$$
\frac{U_{e x}}{k_{B} T N}=-\frac{9}{4}(b \Gamma)^{\frac{3}{2}} \arctan \left(\frac{1}{\sqrt{b \Gamma}}\right)
$$

of the OCP. To obtain Eq.(24) we use the relation $U_{\mathrm{ex}}=\Gamma \partial F_{\mathrm{ex}} / \partial \Gamma$. Again we note that the same functional dependence for the excess internal energy (but with different coefficients) has been obtained in [44].

\section{RESULTS AND DISCUSSION}

As it follows from Eqs.(23) and (24), for $\Gamma \rightarrow 0$ the Debye-Huckel behavior is recovered. On the other hand in the opposite limit $\Gamma \gg 1$ Eqs.(23) and (24) demonstrate the linear behavior on $\Gamma$ with the leading term $-A \Gamma$ in accordance with fits (4) and (5) to the $\mathrm{MC}$ data. The constant $A$ reads

$$
A=\frac{9}{4} b=\frac{3}{2}\left(\frac{2}{\pi^{2}}\right)^{1 / 3}=0.881 \ldots
$$

which is fairly close to the constant $A=0.899 \ldots$ of the fits (4), (5)).

In Fig. 1 the excess internal energy given by Eq.(24) is compared to the Monte Carlo data, taken from Ref. [14,18] for $0.1 \leq \Gamma \leq 1$ and Ref. [6,20] for $\Gamma>1$. Fig.2 shows the relative

error of the analytical expression (24). As it follows from Fig.1 and Fig.2 the equation of state is fairly accurate in the most of range of the plasma parameter. The maximal deviation of the analytical expression from the numerical data occurs at the intermediate values of the plasma parameter, $0.01<\Gamma<1$.

To analyze the reason of the enhanced deviation of the theoretical results from the numerical data at $0.1<\Gamma<0.5$, one can address the small- $\Gamma$ expansion of $U_{\text {ex }}$ [28]. It was observed [28] that in spite of the correct Debye-Huckel limit, this does not reproduce correctly the next-order terms of the Abe expansion (3). This occurs due to limitations of the Gaussian approximation for the effective Hamiltonian. Since all the coefficients of 
the effective Hamiltonian are known, one can go beyond the Gaussian approximation and develop a usual perturbation scheme, based on the Gaussian Hamiltonian.

\section{FIGURES}
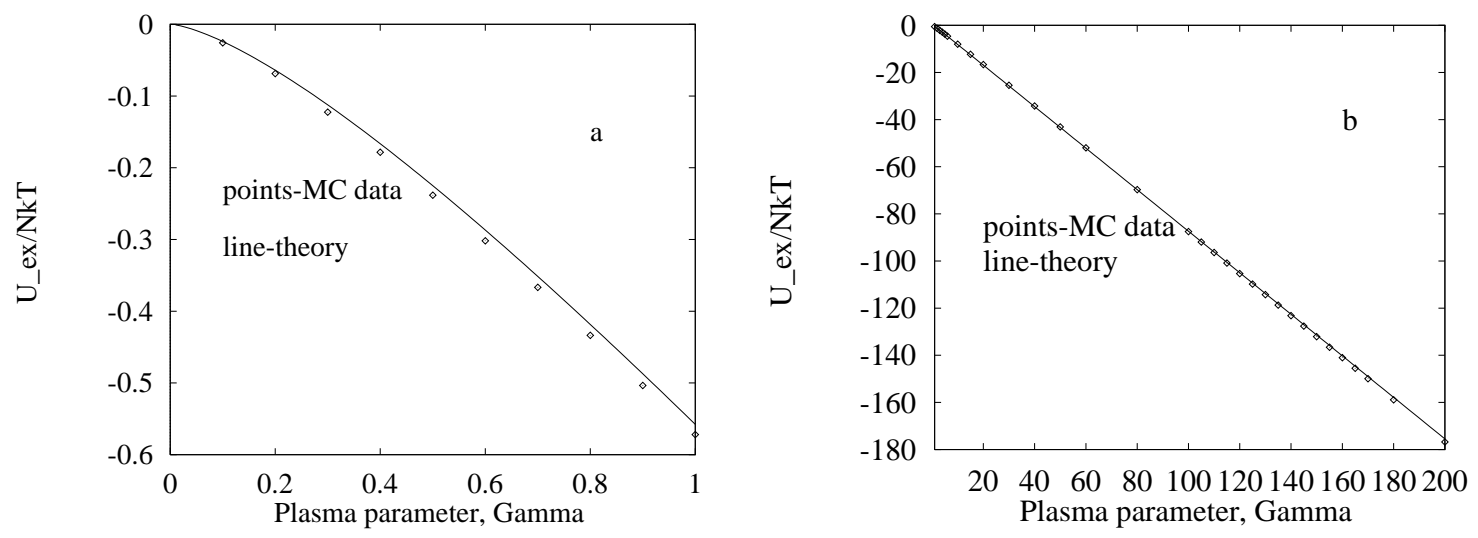

FIG. 1. Shows the dependence of the excess internal energy of the OCP $U_{\text {ex }} / N k_{B} T$ on the plasma parameter $\Gamma=l_{B} / a_{c}\left(l_{B}=e^{2} / k_{B} T, a_{c}^{-3}=4 \pi \rho / 3\right)$. Points give the Monte Carlo data (Ref. $[14,18]$ for $0.1 \leq \Gamma \leq 1$ and Ref. $[6,20]$ for $\Gamma>1$ ); lines represent the theoretical result, Eq. (24)
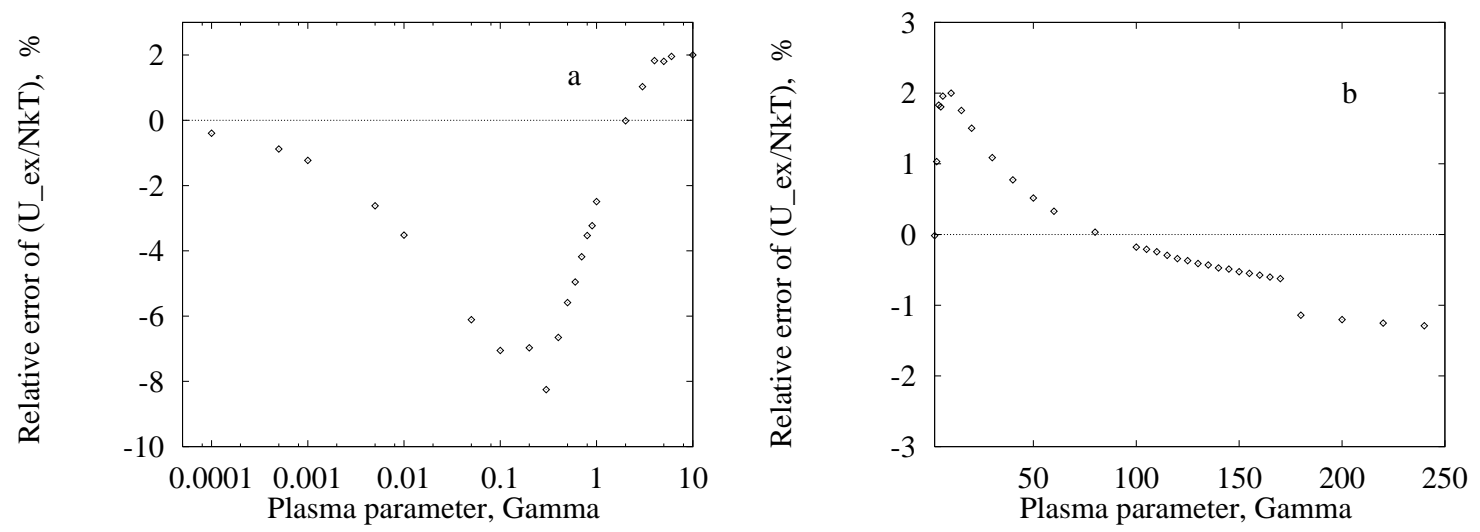

FIG. 2. Shows the relative error (\%) of the analytical expression (24) for the excess internal energy of the OCP $U_{\mathrm{ex}} / N k_{B} T$ as a function of the plasma parameter $\Gamma$. The Monte Carlo data of Ref. [14,18] for $0.1 \leq \Gamma \leq 1$ and of Ref. [6,20] for $\Gamma>1$ are used. For $\Gamma<0.1$ Eq. 24. is compared with the Abe expansion Eq.(3) 
In particular the equation of state in a form of the virial expansion may be recovered [37. Unfortunately this expansion does not provide the closed analytical expression for the excess thermodynamic functions, which may be used with acceptable accuracy for all the range of $\Gamma$.

Thus, dealing with a problem where the relevant range of plasma parameter is not known in advance one should preferably use the simple closed-form equations suggested in the present study.

\section{CONCLUSION}

A "first-principles" equation of state for the one-component plasma is derived that has a correct Debye-Huckel behavior at the limit of small plasma parameter $\Gamma$ and demonstrates a linear dependence on $\Gamma$ at $\Gamma \gg 1$. The obtained coefficient 0.881 at the linear leading term is close to the corresponding coefficient 0.899 found in the Monte Carlo simulations. The simple analytical expression for the excess internal energy reproduces the MC data within $1-2 \%$ accuracy for the most of range of $\Gamma(0 \leq \Gamma \leq 250)$ and has a typical deviation of the order of $2-5 \%$ (with a maximal one $\approx 8 \%$ ) for $0.01<\Gamma<1$.

\section{ACKNOWLEDGEMENTS}

Helpful discussions with John Valleau, George Stell, Werner Ebeling, Yaakov Rosenfeld and Jens Ortner are highly appreciated. The author is thankful for Torsten Kahlbaum for providing some important references. Financial support of NSERC of Canada is acknowledged. 


\section{REFERENCES}

[1] M. Baus and J. P.Hansen Phys.Rep., 591 (1980).

[2] S.Ichimaru, H. Iyetomi and S. Tanaka, Phys.Rep., 14991 (1987).

[3] H. M. Van Horn, in White Dwarfs ed. by W. J. Luyten, (Springer, Berlin, 1971), p.96.

[4] B. Jancovici, J.Stat.Phys., 17357 (1977).

[5] J. P.Hansen Phys.Rev.A, 83096 (1973).

[6] D. A. Young, E. M. Corey and H. E. DeWitt, Phys.Rev. A 44, 6508 (1991).

[7] M. Ross, H. E. DeWitt, and W. B. Hubbard, Phys.Rev. A 24, 1016 (1981); W. H. Young, J.Phys.F: Met.Phys., 12, L19 (1982); N. Montella, G. Senatore, and M. P. Tosi, Physica $B+C, \mathbf{1 2 4}, 22$ (1984); S. Ono, and I. Yokoyama, J.Phys.F: Met.Phys., 14, 2909 (1984); T. Itami, and M. Shimoji, J.Phys.F: Met.Phys., 14, L15 (1984).

[8] A. M. Bratkovsky, J.Phys.C: Cond.Matter, 1, 3453 (1989).

[9] M. J. Stevens, and M. O. Robbins, Europhys.Lett., 12, 81 (1990).

[10] H. Löwen, Phys.Rep., 237, 249 (1994); H. Löwen, P. A. Madden, and J. P. Hansen, Phys.Rev.Lett., 68, 1081 (1992); H. Löwen, J. P. Hansen, and P. A. Madden, J.Chem.Phys. 98, 3275 (1993).

[11] R. Penfold, B. Jonsson, and C. E. Woodward, J.Chem.Phys. 92, (1990); R. Penfold, B. Jonsson, and S. Nordholm, J.Chem.Phys. 99, 497 (1993); R. Penfold, S. Nordholm, B. Jonsson, and C. E. Woodward, J.Chem.Phys. 95, 2048 (1991).

[12] H.E.De Witt, J.Math.Phys., 3, 1216 (1962); G.Kelbg, Ann.Physik, 12, 1216 (1963); H.J.Hoffmann and W.Ebeling, Physica , 39, 593 (1968); C.Deutsch and M.Lavaud, Phys.Lett.A, 39, 253 (1972), 43, 193 (1973); W.Ebeling and A.Föerster, in Condensed Matter and Plasma in Physics and Astronomy, eds. S.Ichimaru and S.Ogata (AddisonWesley, Reading (MA), 1994; H.Lehmann and W.Ebeling, Phys.Rev. E 54, 2451 (1996). 
[13] R.Abe, Progr.Theor.Phys., 22, 213 (1959); see also C.Deutsch, Y.Furutani and M.M.Combert, Phys.Rep., 6985 (1981).

[14] W.L.Slattery, G.D.Doolen and H.E.De Witt, Phys.Rev.A, 212087 (1980).

[15] E.G.D.Cohen and T.J.Murphy, Phys.Fluids, 12, 1404 (1969).

[16] J.F.Springer, M.A.Pokrant and F.A.Stevens, J.Chem.Phys., 58, 4863 (1973); P.Hutchinson and W.R.Conkie, Mol.Phys., 3, 567 (1972); K.C.Ng, J.Chem.Phys., 61, 2680 (1974).

[17] Y.Rosenfeld and N.W.Aschcroft, Phys.Rev.A, 20, 1208 (1979); 2162 (1979); Y.Rosenfeld, Phys.Rev.Lett., 20, 1208 (1980); Y.Rosenfeld and W.Gelbart J.Chem.Phys., 61, 2680 (1985).

[18] A.A.Broyles, H.L.Sahlin and D.D.Carley Phys.Rev.Lett., 10, 319 (1963).

[19] S.G.Brush, H.L.Sahlin and E.Teller, J.Chem.Phys., 45, 2102 (1966).

[20] W.L.Slattery, G.D.Doolen and H.E.De Witt, Phys.Rev.A, 262255 (1982).

[21] G.S.Stringfellow, H.E.De Witt and W.L.Slattery, Phys.Rev.A, 411105 (1990).

[22] R.T.Farouki and S.Hamaguchi, Phys.Rev.E, 474330 (1993).

[23] H.E.De Witt, W.L.Slattery and G.S.Stringfellow, in Strongly Coupled Plasma Physics, ed. S.Ichimaru (Elsevier, Amsterdam, 1990), p.635. see also H.E.De Witt, Contr.Plasma Phys. 33, 399 (1993).

[24] B.Brami, J.P.Hansen and F.Joly, Physica A 95, 505 (1979).

[25] T.Kahlbaum, in Physics of Strongly Coupled Plasma, ed. W.D.Kraeft and M.Schlanges (World Scientific, 1996), p.99.

[26] H.E.De Witt and Y.Rosenfeld, Phys.Lett.A, 75, 79 (1979).

[27] S.Nordholm, Chem.Phys.Lett., 105, 302 (1984). 
[28] W.Ebeling, On the Thermodynamic Functions of the One-Component Nonideal Plasma, preprint.

[29] The term with $\mathbf{k}=0$ conceals with the terms describing the self-interaction of the compensating background and interaction of the background with particles.

[30] J. Hubbard, and P. Schofield, Phys. Lett., A40, 245 (1972).

[31] N.V.Brilliantov and J.P.Valleau, J.Chem.Phys., 108, 1123 (1998); N.V.Brilliantov, Phys.Rev. E, in press.

[32] To obtain Eq.(9) we take into account that $\rho_{\mathbf{k}} \rho_{-\mathbf{k}}=\left(\rho_{\mathbf{k}}^{c}\right)^{2}+\left(\rho_{\mathbf{k}}^{s}\right)^{2}$ where $\rho_{\mathbf{k}}^{c}$ and $\rho_{\mathbf{k}}^{s}$ are respectively the real (cosine) and imaginary (sine) parts of of $\rho_{\mathbf{k}}$. Similar relation for $\varphi_{\mathbf{k}} \varphi_{-\mathbf{k}}$ is used.

[33] R. Kubo, J.Phys.Soc. Jap., 17, 1100 (1962).

[34] N.Goldenfeld, Lectures on Phase Transitions and the Renormalization Group, AddisonWesley Publishing Company, NY, 1992; S.-K. Ma, Modern Theory of Critical Phenomena (Benjamin, New York, 1976).

[35] The ideal-gas cumulants, Eq.(11), may be easily computed using the standard technique; in application to fluids, see, e.g. T. Morita, and K. Hiroike, Progr.Theor.Phys., 25, 537 (1961), or I. R. Yukhnovskii and M. F. Golovko, Statistical Theory of Classical Equilibrium Systems, (in Russian) (Naukova Dumka, Kiev, 1980), where these are explicitely given.

[36] A.L. Kholodenko, A.L.Beyerlein, Phys.Rev. A, 34, 3309 (1986).

[37] J. Ortner, private communication.

[38] J. Glimm and A. Jaffe, Quantum Physics (Springer-Verlag, New York, 1981).

[39] N. V. Brilliantov, C. Bagnuls, and C. Bervillier, Phy.Lett.A, in press. 
[40] The self-energy is equal to the energy of interactions of two charges at vanishing distance. This divergency does not affect the final result and may be removed considering the lattice version of the problem 36, 38, or (equivalently) implying an infinitesimally small hard-core for interacting particles.

[41] N. Krall and A. Trivelpiece, Principles of Plasma Physics (McGraw-Hill, New York, 1973).

[42] J.M.Ziman, Principles of the Theory of Solids. (Clarendon Press, Oxford, 1964).

[43] Note that the restriction $\varphi_{-\mathbf{k}}=\varphi_{\mathbf{k}}^{*}$ does not reduce the total number of physically different modes, since the modes with the wave-vectors $\mathbf{k}$ and $\mathbf{- k}$ correspond to that spreading in the opposite directions.

[44] A.S.Kakljugin, Teplofizika Visokih Temperatur, 23, 217 (1985). 